Ephemeride für $\mathbf{2}^{\text {h }}$ M. Z. Berlin.

\begin{tabular}{|c|c|c|c|c|c|c|}
\hline $189 \mathrm{I}$ & $c$ & & & $\delta$ & $\log \Delta$ & Ab. Zt. \\
\hline Sept. 23 & $I^{\mathrm{h}} 29^{\mathrm{s}}$ & $8: 2$ & +5 & $44: 8$ & $0.3^{1} 3^{68}$ & $17^{\mathrm{m}} 7^{5}$ \\
\hline 24 & 28 & 25.0 & & 44.0 & & \\
\hline 25 & 27 & 40.8 & 5 & 432 & & \\
\hline 26 & 26 & 55.6 & & $4^{2 \cdot 3}$ & & \\
\hline 27 & 26 & 9.6 & 5 & $4^{1} \cdot 3$ & 0.30891 & 1656 \\
\hline 28 & 25 & 22.7 & & 40.3 & & \\
\hline 29 & 24 & 35.1 & & $39 \cdot 3$ & & \\
\hline $3^{\circ}$ & 23 & 46.7 & & $3^{8.2}$ & & \\
\hline Oct. $\quad \mathbf{r}$ & 22 & 57.6 & & 37.1 & $0.3 \circ 5 \circ 3$ & 1647 \\
\hline 2 & 22 & 8.0 & & 35.9 & & \\
\hline 3 & 21 & 17.8 & & 34.8 & & \\
\hline 4 & 20 & 27.0 & & 33.6 & & \\
\hline 5 & I 9 & 35.8 & & 32.4 & 0.30210 & 1640 \\
\hline 6 & 18 & 44. I & & $3^{1.2}$ & & \\
\hline 7 & 17 & 52.1 & & 30.0 & & \\
\hline 8 & 16 & 59.7. & 5 & 28.7 & & \\
\hline 9 & I 6 & 7.1 & 5 & 27.5 & 0.30013 & r6 35 \\
\hline 10 & 15 & 14.3 & & 26.3 & & \\
\hline I I & 14 & $21 \cdot 3$ & 5 & 25.1 & & \\
\hline 12 & I 3 & 28.3 & 5 & 23.9 & & \\
\hline 13 & I I 2 & 35.2 & +5 & 22.7 & 0.29917 & 1633 \\
\hline
\end{tabular}

\begin{tabular}{|c|c|c|c|c|c|c|}
\hline $189 \mathrm{I}$ & \multicolumn{2}{|c|}{$\alpha$} & \multicolumn{2}{|c|}{$d$} & $\log \Delta$ & Ab. Zt. \\
\hline Oct. $\mathbf{I}_{3}$ & $I^{\mathrm{h}} \mathrm{I}_{2}$ & $35^{5.2}$ & $+5^{\circ}$ & $22: 7$ & $0.299 \times 7$ & $16^{\mathrm{m}} 33^{\mathrm{s}}$ \\
\hline 14 & 11 & 42.0 & & 21.6 & & \\
\hline 15 & 10 & 49.0 & & 20.4 & & \\
\hline 16 & & 56.0 & & I 9.3 & & \\
\hline 17 & & $3 \cdot 2$ & & I 8.2 & $0.299^{2} 3$ & 1633 \\
\hline 18 & & 10.7 & 5 & I 7.1 & & \\
\hline 19 & & I 8.4 & 5 & I 6.1 & & \\
\hline 20 & & 26.4 & 5 & I 5.1 & & \\
\hline 21 & & 34.7 & 5 & 14.2 & 0.30033 & $163^{6}$ \\
\hline 22 & & 43.5 & 5 & I $3 \cdot 3$ & & \\
\hline 23 & & $5^{2.8}$ & 5 & 12.5 & & \\
\hline 24 & & 2.6 & 5 & I 1.7 & & \\
\hline 25 & & I 2.9 & 5 & 10.9 & 0.30241 & 1640 \\
\hline 26 & I & 23.8 & 5 & 10.2 & & \\
\hline 27 & I 0 & $35 \cdot 4$ & 5 & 9.6 & & \\
\hline 28 & $\circ 59$ & 47.8 & 5 & 9.1 & & \\
\hline 29 & 59 & 1.0 & 5 & 8.6 & 0.30545 & 1648 \\
\hline $3^{\circ}$ & $5^{8}$ & 15.0 & 5 & 8.2 & & \\
\hline $3 I$ & 57 & 29.9 & 5 & 7.8 & & \\
\hline Nov. I & 56 & $45 \cdot 7$ & 5 & 7.6 & & \\
\hline 2 & $\circ 56$ & 2.5 & +5 & 7.4 & $0.3093^{8}$ & I6 57 \\
\hline
\end{tabular}

Bonn 189 I Aug. 10.

Opposition in AR. 1891 Oct. 13 ; Grösse 115 .

\title{
Entdeckung eines Planeten durch Herrn Dr. J. Palisa in Wien.
}

\section{(Telegramm.)}

Planet zwölfter Grösse I4 August $12^{\mathrm{h}} 5^{8^{\mathrm{m}}} \cdot 3$ M. Z. Wien AR. $=349^{\circ} 57^{\prime} 39^{\prime \prime} ;$ PD. $=94^{\circ} 1^{\prime} 3^{\prime \prime}$.

Bew. in $\mathrm{AR} .=-10^{\circ}$, in $\mathrm{PD} .=+4^{\prime}$.

Das Telegramm, welches hier am I 5. August einlief, wurde an die Mitglieder der Centralstelle sofort weitergegeben.

Von diesem Planeten theilt Herr Dr. Palisa brieflich die folgenden genauen Beobachtungen mit:

$$
\text { Aug. } 2412^{\mathrm{h}} 5^{8^{\mathrm{m}}} 28^{\mathrm{s}}
$$

* Rümk. $11108 \quad 23^{\mathrm{h}} 18^{\mathrm{m}} 27^{\mathrm{s}} \cdot 43-4^{\mathrm{o}} 14^{\prime} 4^{\prime \prime} .9$

$$
\begin{array}{rrrr} 
& +2.21 & & +13.1 \\
+120.93 & & -10.5 \\
\hline 2319 & 50.57 & -4 & 153^{6.3} \\
& 8.326 & & 0.844
\end{array}
$$

$$
\begin{aligned}
& \text { Aug. } 15{ }_{10}^{\mathrm{h}}{ }_{1} 8^{\mathrm{m}_{1}}{ }^{\mathrm{s}} \\
& 23^{\mathrm{h}} \text { I } 8^{\mathrm{m}} 27^{\mathrm{s}} \cdot 43-4^{\circ} \text { I } 4^{\prime} 4^{8} \text {."9 } \\
& +2.23+13.2 \\
& \begin{array}{rrr}
+49.64 & -449.7 \\
1919.30 & -41925.4
\end{array} \\
& 9.489 \mathrm{n} \quad 0.835
\end{aligned}
$$

Daraus die tägliche Bewegung von $-35 \cdot 5-4: 3$.

F. Palisa.

Herr Berberich hält die Identität des Planeten entweder mit (149) Medusa oder mit (291) Alice der ähnlichen Bahnlage wegen für nicht unmöglich; er hat seine Untersuchungen darüber noch nicht abgeschlossen. Die fruheren Elemente dieser Planeten geben freilich die Bewegung in AR. zu etwa $-40^{\mathrm{s}}$, also wohl mit dem Telegramm, aber nicht mit den genaueren Wiener Beobachtungen übereinstimmend. - Sollte der Planet neu sein, so würde er die Nummer (3 I 2) erhalten.

Kiel 1891 Aug. 21.

$$
L \text {. }
$$

Inhalt zu Nr. 3055. Th. Albrecht. Resultate der fortgesetzlen Beobaclitungsreilen in Berlin und Prag betreffend die Veränderlichkeit der Polhöhe. I29. - Dubiago. Beobachtungen auf der Sternwarte in Kasan. 135. - G. Marczell. Sonnenfinsterniss I891 Juni 6. 135. - G. Lezwitzky. Beobaclitungen der totalen Mondfinsterniss 1891 Mai 23. 137. -- L. Weinek. Neue Rille und neuer Krater, entdeckt auf den Mondphotographien der Lick Sternwarte. 139. - C. Mönnichmeyer. Elemente und Ephemeride des Planeten (250) Bettina. 141. - Entdeckung eines Planeten durch Herrn Dr. Palisa in Wien. I43. 